\section{ZINC PROTAMINE INSULIN}

\section{A CLINICAL TRIAL OF THE NEW PREPARATION} BY

\author{
R. D. LAWRENCE, M.D., F.R.C.P. \\ AND
}

NORA ARCHER, M.B.Oxon.*

(From the Diabetic Clinic, King's College Hospital)

In 1936 Hagedorn and his Danish colleagues introduced to the world a new insulin compound, protamine insulin. They had already employed it therapeutically in Denmark for some two years, and it has been on the market in this country for six months under the name Leo "insulin retard." The uses and advantages of this protamine insulin have been described in many articles published in English and American medical journals (Hagedorn, 1936 ; Joslin et al., 1936 ; Krarup, 1935 ; Lawrence and Archer, 1936; Rabinowitch et al., 1936a ; Root et al., 1936; Wilder, 1936).

Protamine is a simple protein obtained from the sperm of fishes. At $p \mathrm{H} \mathrm{7.2} \mathrm{it} \mathrm{forms} \mathrm{with} \mathrm{insulin} \mathrm{a} \mathrm{pre-}$ cipitated compound, protamine insulin, which is relatively insoluble in the subcutaneous tissues, and is therefore more slowly absorbed than is the soluble insulin hydrochloride in general use. The latter is so rapidly absorbed that its action is finished quickly, making two or three injections a day necessary for good control of severe diabetes. As many as four and five injections a day have been administered by some workers. Protamine insulin certainly acts for twelve hours, so that it has been possible to ensure a normal fasting blood-sugar level in the morning by giving an adequate dose of this compound in the evening. Hagedorn recommended that it should be used in this way, with soluble insulin in the morning to act quickly enough to control the sugar resulting from digestion of the day's carbohydrate food. We felt, with many others, that an attempt should be made for the patient's sake to control diabetes with a single daily dose of protamine insulin. The results were good with mild cases, but even with very large doses-60 to 100 unitscontrol was seldom possible with severe cases. Fortunately this possibility has been more nearly realized by the discovery of a longer-acting and more stable compound, zinc protamine insulin.

This paper describes the known actions and qualities of the new preparation and the clinical results we have obtained in forty-two patients who could not be controlled by diet alone, and required insulin. The number of cases is small, but the majority have been carefully observed under hospital conditions, and the results have been sufficiently consistent for us to form an opinion on the use, advantages, and disadvantages of zinc protamine insulin. We have also had the privilege of seeing it employed in several Canadian and American clinics, where experimental work was started with it in June, 1936.

\section{Nomenclature}

We have felt, since working with more than one type of insulin, and more especially since trying to write about them, that some accepted code of nomenclature is essential to avoid confusion.

Until a year ago " insulin" was insulin, and meant only one thing, at least clinically. Since then protamine insulin has come from Denmark and zinc protamine insulin from

* Research Scholar, British Medical Association.

Toronto, and it seems likely that improved modifications of these may appear at any moment to add to the confusion of medical nomenclature and thought. The fundamental difference between the "old," "regular," or " ordinary" insulin (but surely insulin will always remain extraordinary, at least to the diabetic) and the new longaction insulins is their solubility at the $p \mathrm{H}$ at which they are injected. The old insulin is injected in solution; the new insulips are injected precipitated in a suspension form. We propose to use these terms to distinguish between them. Below are some of the synonyms that are already current :

I. Insulin hydrochloride in solution in weak acid, $p \mathrm{H} \mathrm{3-4:}$ $\begin{array}{ll}\text { Old insulin. } & \text { Quick-acting insulin. } \\ \text { Ordinary insulin. } & \text { Soluble insulin. } \\ \text { Regular insulin. } & \end{array}$

II. Precipitated compounds in suspension at neutral reaction, $p \mathrm{H}$ 7.2: Suspension Insulin

Protamine Insulin

Leo "insulin retard" (commercial name)

Danish protamine insulin
Protamine insulinate

$$
\begin{aligned}
& \text { Zinc Protamine Insulin } \\
& \text { Protamine zinc insulin } \\
& \text { Canadian protamine insulin } \\
& \text { Zinc protamine insulin sus- } \\
& \text { pension } \\
& \text { Protamine insulin (with } \\
& \text { zinc) suspension. (Form } \\
& \text { adjusted by the Ministry } \\
& \text { of Health and recently } \\
& \text { adopted by insulin manu- } \\
& \text { facturers) }
\end{aligned}
$$

When we remember that not only the medical profession but also the diabetic public buy and use these products, the necessity for a clear distinction between them is obvious, particularly in the naming and labelling of commercial preparations. In the past the preparations of soluble insulin sold by different manufacturers have been almost indistinguishable in chemical nature and certainty in clinical action. We hope that steps will be taken to make the new preparations, such as zinc prot:mine insulin, as uniform as possible in zinc and protamine content and in the type of fish protamine employed.

\section{Zinc Protamine Insulin}

The discoverer of this compound is D. A. Scott of Toronto, who had already worked on zinc insulin compounds for several years and had shown that crystalline insulin was a true chemical compound of zinc and insulin, zinc insulinate (Scott and Fisher, 1935). When he turned his attention to protamine insulin compounds he found that when entirely freed from traces of zinc and allied metals they were no more prolonged in action than the soluble insulin hydrochloride (Fisher and Scott, 1935). The addition of traces of zinc and the adjustment of the $p \mathrm{H}$ to 7.2 produced a zinc protamine insulin compound in suspension which was more stable and more prolonged in its action than any previously discovered. This has been proved experimentally (Fisher and Scott, 1935; Rabinowitch et al., 1936b) both in men and animals, and we find the length of action roughly proportional to the size of dose. The exact chemical nature of the precipitated compound-whether a true salt or merely an indeterminate grouping-is not known. Fortunately it is sufficiently stable to be issued in suspension in one bottle ready for injection. This is an important advance on previous protamine compounds, which had to be issued in two bottles-the buffer separate from the protamine insulin -and mixed by the patient before use. The new compound is a stable fine suspension which sediments on standing; the bottle therefore requires shaking before injection to ensure a uniform suspension. So far only one strength, 40 units per c.cm., has been prepared, but we think 80 units per 
c.cm. will also be necessary for the convenience of patients.

The amount of zinc added is very small $-1 \mathrm{mg}$. in 500 units of insulin-so that even the severest diabetic would not receive more than 1 gramme of zinc in ten years. Zinc in this substance is not toxic, and has not caused any general ill effects.

\section{Action of Zinc Protamine Insulin}

The difference in action between soluble insulin and zinc protamine insulin seems to be entirely due to the difference in their rates of absorption from the subcutaneous tissues. Soluble insulin begins to lower the blood sugar from fifteen to thirty minutes after the time of injection. Zinc protamine insulin shows little, if any, effect on the blocd sugar in the first three to six hours after injection. The action of soluble insulin rarely lasts longer than eight to ten hours, however large the dose. Zinc protamine insulin continues its action for twenty-four hours when administered in quite moderate doses from 20 to 24 units; in larger doses it will continue to act even longer. The fall in the blood sugar from zinc protamine insulin is more gradual than from soluble insulin, so that severe hypoglycaemia cannot occur soon after the injection, and the violent oscillations between hyper- and hypo-glycaemia sometimes experienced by severe diabetics on soluble insulin are eliminated. These qualities are advantages.

On the other hand, one obvious drawback arises from the slow absorption of zinc protamine insulin. It does not enter the circulation rapidly enough to deal with sugar absorbed into the blood stream from a meal containing 40 or more grammes of carbohydrate. Thus it is usual in a severe diabetic on a single morning dose of zinc protamine insulin to find blood sugars of 180 to $250 \mathrm{mg}$. per $100 \mathrm{c.cm}$. of blood soon after food, while a few hours later the blood sugar is normal. There is no return of ketosis with this hyperglycaemia after food. Another disadvantage we have noted is considerable irregularity in action from day to day. This is due, we suppose, to differences in the dispersion of the injections and in the vascularity, of the surrounding tissues, so that the precipitate goes into solution and is absorbed at different rates. The action of soluble insulin is much more regular from day to day, no doubt because it enters the general circulation so rapidly and completely.

It seems clear that the normal secretion and action of insulin is not fully imitated either by the soluble or suspension insulins. The probability is (Lawrence and Archer, 1936) that a constant small secretion of insulin from the normal pancreas keeps the endogenous sugar under control, while an added intermittent secretion deals with the exogenous sugar absorbed from carbohydrate digestion. The steady, slow action of zinc protamine insulin imitates the small constant secretion, but cannot control hyperglycaemia after much carbohydrate food. On the contrary, soluble insulin acts strongly and well when given balanced by carbohydrate, but, because of its evanescent action, quickly allows endogenous hyperglycaemia to recur.

A combination of the two types of insulin action is needed to turn the severe diabetic into a physiological individual, and that has not yet been achieved by any single preparation. The next best thing, however, is to mix soluble and suspension insulin in the correct proportions and give them at one injection, so that the soluble insulin controls the post-prandial hyperglycaemia while the suspension insulin comes into action later in the day and keeps the blood sugar normal throughout the night. So far a mixture of soluble and suspension insulin in one bottle has not been produced, but we have satisfied ourselves by clinical experiments that soluble and suspension insulin can be given in the same syringe and injected into the same place and each retains its characteristic action (see table).

\begin{tabular}{|c|c|c|c|c|c|}
\hline \multirow{3}{*}{$\begin{array}{c}\text { Time } \\
8 \text { a.m. } \\
10 \text { a.m. }\end{array}$} & \multicolumn{2}{|c|}{$\begin{array}{c}\text { Case I } \\
\text { Blood Sugar } \\
\text { (mg. per } 100 \text { c.cm. blood) }\end{array}$} & \multicolumn{3}{|c|}{$\begin{array}{c}\text { Case II } \\
\text { Blood Sugar } \\
\text { (mg. per } 100 \text { c.cm. blood) }\end{array}$} \\
\hline & 82 & 116 & 272 & 274 & 347 \\
\hline & -117 & 209 & & & \\
\hline Noon & 92 & 209 & 126 & 187 & 333 \\
\hline 2 p.m. & & & 183 & 150 & \\
\hline 4 p.m. & 81 & & 228 & 88 & \\
\hline $\begin{array}{l}\text { Insulin } \\
\text { given } \\
8 \text { a.m. }\end{array}$ & $\begin{array}{l}20 \text { units } \\
\text { soluble }+ \\
30 \text { units zinc } \\
\text { protamine } \\
\text { insulin }\end{array}$ & $\begin{array}{l}30 \text { units zinc } \\
\text { protamine } \\
\text { insulin }\end{array}$ & $\begin{array}{l}30 \text { units } \\
\text { soluble } \\
\text { insulin }\end{array}$ & $\begin{array}{l}30 \text { units } \\
\text { soluble }+ \\
30 \text { units zinc } \\
\text { protamine } \\
\text { insulin }\end{array}$ & $\begin{array}{c}30 \text { units zinc } \\
\text { protamine } \\
\text { insulin }\end{array}$ \\
\hline
\end{tabular}

Breakfast, 9 a.m., 50 grammes carbohydrate ; lunch, 1 p.m., 30 grammcs carbohydrate.

\section{Clinical Use of Zinc Protamine Insulin}

We are convinced that zinc protamine insulin has passed the experimental stages and is ready for therapeutic application. We are sure that one injection a day will maintain all but the most exceptionally severe diabetics in good health; but we are equally certain that we have not had enough experience to lay down hard-andfast rules for its prescription. We cannot do more than indicate how we have used it and the results we have obtained. As with soluble insulin, it is essential to study each case individually to find the optimum dose and diet balance. We should like to point out, too, that suggestions which seem sound to us from four months' experience may have to be considerably revised in the light of further studies.

With the exception of one patient-who required 500 units a day of soluble insulin and is now stable on 90 units twice a day of zinc protamine insulin-all our patients on zinc protamine have been treated with one injection a day. Since the action of suspension insulin is cumulative, three to six days must elapse before the true effect of any constant daily dose can be assessed.

\section{The Basal Dose}

The first step is to discover what we have called the basal dose of insulin-that which will continue its action for twenty-four hours. The correct dose is that which will cause no hypoglycaemia during the night and yet maintain the blood sugar at a normal level before breakfast next morning. We have given 30 units before breakfast as a starting dose, and adjusted it up or down according to blood and urine tests.

Where fasting blood-sugar estimations are not available two specimens of urine should be taken before breakfast: one immediately on waking, to void any sugar which may have entered the bladder soon after bedtime; and a second just before breakfast, to indicate the approximate level of the blood sugar at that time. Even when the first specimen contains sugar the second is often sugarfree, indicating that the dose of zinc protamine insulin is sufficient to last the twenty-four hours. If the second specimen contains sugar the dose must be raised. If there are any symptoms of hypoglycaemia-sweating, palpitations, and trembling between midnight and breakfastthe basal dose is too high and must be reduced. We have 
found in many cases this basal dose to be 20 to 30 units; in the more severe, 30 to 50 units.

In the course of establishing the patient on the basal dose of zinc protamine insulin it becomes apparent to which type of diabetic he belongs. The milder insulin case remains sugar-free throughout most of the day on his basal dose of suspension insulin. Sometimes there is slight hyperglycaemia in the early forenoon, a time when the injection of the previous day is exhausted and that of the same day not established. Fig. 1 shows a series of blood-sugar estimations on such a case throughout the day.

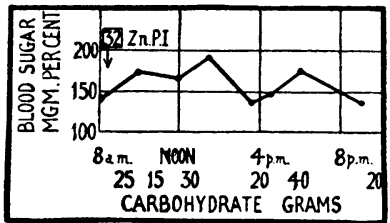

FIg. 1.-Blood-sugar curve after twenty-one days on zinc protamine insulin.

\section{The Addition of Soluble Insulin}

The severe type of diabetic cannot be kept well controlled throughout the twenty-four hours by a single dose of suspension insulin in the morning, and tends to pass large amounts of sugar, but not acetone bodies, after each meal. This cannot be overcome by increasing the basal dose, because hypoglycaemia is certain to appear between bedtime and breakfast. It can be mitigated by cutting down breakfast and lunch carbohydrate to a very low level, but this is undesirable and the patients naturally dislike it. The obvious treatment is to give soluble insulin along with the basal dose of zinc protamine insulin, and fortunately we have found that they can be mixed in one syringe and injected together, and yet retain their separate qualities of action (see table and Fig. 2).

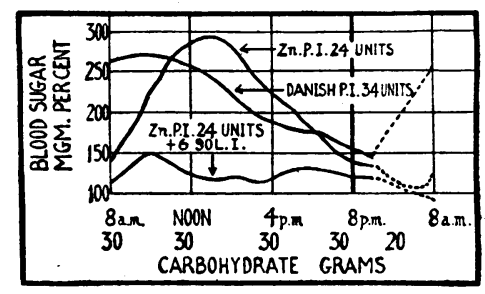

FIG. 2 shows the effect of different insulins given at 8 a.m. Zinc protamine insulin 24 units acts longer than Danish protamine insulin 34 units, but controls the day's carbohydrate food poorly. The addition of soluble insulin rectifies this.

If the urine is sugar-free before breakfast a mixed dose should be administered almost immediately before food, as a longer interval may result in hypoglycaemia before the carbohydrate is absorbed. Another plan we have tried is to inject the basal dose at bedtime instead of before breakfast. In this way its minimum action coincides with the hours of sleep and its maximum with the intake of the day's food. This has worked satisfactorily in six patients; one patient had slight hypoglycaemia at 5 a.m. on a dose of 30 units at 10 p.m. When finally stabilized her basal dose proved to be 14 units. The blood sugar is generally controlled for about eighteen to twenty hours, but the evening's carbohydrate meals lead to heavy glycosuria. We think that most patients will be better controlled when given suspension insulin before breakfast rather than at bedtime. When it is necessary to add soluble insulin to the basal dose this must be taken before breakfast; the mixed dose administered at night would certainly result in hypoglycaemia.

\section{Diet and its Arrangement}

It has become the general practice to allow liberal amounts of carbohydrate in the diet of diabetics on insulin. Diets containing 120 to 200 grammes of carbohydrate can be tolerated very satisfactorily on zinc protamine insulin, but the carbohydrate distribution must be carefully arranged. It is customary with soluble insulin to order most carbohydrate at the two meals which follow the morning and evening injections, 150 grammes (g.) being taken as :

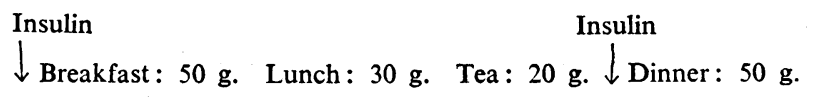

The action of a basal dose of zinc protamine insulin is slow in beginning, gathers strength, acts for twenty-four hours, and may produce hypoglycaemia any time when no carbohydrate has been taken for three to five hours. Therefore the carbohydrate must be spread throughout the day in smaller and more frequent feeds than were advised before. We consider an afternoon tea and a bedtime feed essential to prevent hypoglycaemia. A suitable arrangement of 150 grammes would be:

Insulin

$\downarrow$ Breakfast : 30 g. Lunch: 30 g. Tea: 30 g. Dinner: 40 g.

In severe cases, where soluble is added to suspension insulin, more carbohydrate should be given at breakfasttime-for example, 50 grammes instead of 30 grammes. Variations in the amount of carbohydrate at each meal should be made in each individual case to strike the best balance between glycosuria and hypoglycaemia.

\section{Hypoglycaemia}

Soluble insulin exerts its main effect from three to six hours after injection and therefore its overdoses occui between these times. The onset of hypoglycaemia from zinc protamine insulin is quite different, coming much later in the day. Both have a later hypoglycaemic action when administered in large doses. We have found that 15 to 25 units of zinc protamine insulin act most strongly eight to twelve hours after injection, a morning dose causing hypoglycaemia before tea or before dinner; while 25 to 30 units act most strongly fourteen to twenty-two hours after injection, causing hypoglycaemia during the early hours of the morning, or even just before breakfast next day.

Hypoglycaemia is more subtle in onset, and on the whole subjective symptoms are less severe than with soluble insulin, but we believe that the sense of security produced by these observations is a false one. Preliminary symptoms of shakiness, sweating, and palpitation, usually ascribed to an adrenaline reaction, may be absent when the blood sugar is 40 to $60 \mathrm{mg}$. per $100 \mathrm{c.cm}$. of blood. On the few occasions that we have been able to take blood for sugar estimation when symptoms of hypoglycaemia were actually present, the blood sugar has been exceedingly low. It was noticed that a patient was sweating and trembling a little when blood was taken from his ear one evening. He said he noticed nothing himself except feeling rather cold. The blood-sugar concentration was $39 \mathrm{mg}$. per $100 \mathrm{c.cm}$. of blood. Thus the patient is liable to develop severe hypoglycaemic symptoms without warning. This has happened to four of our patients at home, although never under hospital conditions. The new treatment of these four was being worked out without admission to hospital, because this had seemed justifiable in view of their knowledge and experience as trained 
diabetics. The experience has warned us against changes to the new insulin except under close observation. The possibility of severe hypoglycaemia is certainly not eliminated with suspension insulin.

\section{Beginning Treatment in New and Old Cases}

It must be remembered that zinc protamine insulin is slow in coming into action, and a constant dose does not show its full cumulative effect for three to six days. It is advisable, therefore, at the beginning to prescribe soluble insulin as well-at all events if we wish to assess the final dosage quickly in hospital or a nursing home.

In new untreated cases loaded with sugar and acetone we give a moderate dose of soluble insulin, 10 to 20 units, either before the evening meal or with the morning basal dose of 30 units of zinc protamine insulin, and sometimes in severe cases at both times to bring the sugar quickly under control. These added doses of soluble insulin are diminished in two to four days, when the full effect of the basal dose is established. Soluble insulin is omitted altogether in the milder cases, but will be necessary permanently in the more severe types, and is administered in the morning to assist the basal dose of suspension insulin.

In transferring old insulin cases to one dose of zinc protamine insulin the continuation of some soluble insulin for two or three days is necessary if a heavy relapse of sugar is to be avoided. Usually we try as a basal dose three-quarters of the previous total dose of soluble insulin, seldom more than 40 units, with the addition of half the previous morning dose of soluble insulin. The latter addition will gradually be adjusted, reduced, or omitted as the basal dose comes into full action. We must repeat, however, that every case should be watched closely at this stage and judged by the results of urine and blood tests. With our meagre experience we cannot lay down any definite rules for making the change quickly which may not either produce unnecessary hypoglycaemia or allow too severe a relapse in the diabetes. We feel, and experiences from America agree, that the change in severe insulin cases should be made under close observation to avoid danger and to get the best results.

\section{Comparative Dosage of New and Old Insulin}

When large doses of soluble insulin are given to severe diabetics much is wasted, either by destruction in the tissues or more probably by excretion in the urine. The wastage of the slowly absorbed suspension insulin is much less. The basal dose is likely to be at most only some 75 per cent. of the total previous insulin dosage; sometimes we have found it considerably less. The addition of small amounts of soluble insulin necessary in some cases is still likely to leave the total number of units less in the new than in the old treatment. We have had to use far smaller doses of zinc protamine insulin than of Leo "insulin retard" in cases which we tried to control on one big morning dose alone.

\section{Insulin in Dabetic Coma}

Urgent clinical ketosis demands rapid insulin action, and for this reason soluble insulin is often given intravenously to patients in diabetic coma. Whether zinc protamine insulin intravenously is as efficacious we do not yet know; until this is established the old methods of treatment must stand as best.

We have found that zinc protamine insulin is very useful in the treatment of diabetic coma when injected subcutaneously in addition to intravenous or subcutaneous soluble insulin. The suspension insulin administered at the beginning of treatment comes into action six or eight hours later, maintaining, by checking any recurrence of hyperglycaemia, the improvement already secured by the soluble insulin.

\section{General Observations}

New severe cases stabilized on one injection daily of zinc protamine insulin show the same marked clinical improvement as similar cases treated with several injections daily of soluble insulin. Ketosis and all acute symptoms of diabetes disappear within two or three days ; glycosuria and hyperglycaemia more slowly. The usual rapid gain in weight of four to ten pounds is seen in the first week, and energy and health are rapidly restored. Those who wish it return to their ordinary life and full occupation in two to three weeks. As has been the case before, such newly treated diabetics gain considerable carbohydrate tolerance, and the insulin can be reduced in the following weeks.

A striking feature is complete absence of ketosis throughout the twenty-four hours, much more complete than we have ever obtained by three doses of soluble insulin in severe cases of diabetes. Even during the period of hyperglycaemia and glycosuria after a meal there is nc recurrence of ketonuria as judged by the sensitive nitro. prusside test. It appears that the worst defect of diabetics the endogenous production of new sugar and acetone bodies, is incomparably better controlled than ever before

Exercise raises important considerations in the use of the new insulin. It is well recognized that unusual exercise makes diabetics on soluble insulin more liable to hypoglycaemia, but this is limited to the time of its maximum action, which can be foreseen and avoided. Suspension insulin, however, with its prolonged action, will make unusual exercise apt to produce hypoglycaemia at almost any time of the day, and this should be prevented by giving extra carbohydrate before or during exercise. This is more rational than a reduction of the basal dose, because an adequate reduction of the latter would permit recurrent hyperglycaemia next morning. So far we have had no difficulties with patients leading an active life, one being a strenuous engineer mechanic and another playing football one afternoon every: week.

As usual, new cases coming for treatment soon after onset respond best and are most easily managed. Severe diabetics who have already received soluble insulin over a long period require close study during the change-over to zinc protamine insulin. In two such cases treated as out-patients the new treatment had to be stopped on account of irregular action of suspension insulin, which allowed heavy glycosuria on some days and severe hypoglycaemia on others for no obvious reason.

We would hesitate to change to suspension insulin the large number of patients who are well controlled in perfect health and do not mind two injections a day of the soluble insulin. This applies especially to those whose conditions of life are very irregular, as they would be exposed to the danger of hypoglycaemia whenever they missed a carbohydrate meal necessary to balance the long action of zinc protamine insulin. We would remind readers of the irregularity of action from day to day which zinc protamine insulin as well as Danish protamine insulin shows when compared with soluble insulin. On any one day tests which are better or worse than the average may be obtained, and caution in raising or lowering the basal dose on one day's testing should be observed.

From what we have seen of the long action of zinc protamine insulin it seems unlikely that a still longer- 
acting insulin-to be injected, let us suppose, once a week - could ever be successful ; it would cause hypoglycaemia every night, and the patient's condition would be comparable to the rare syndrome of spontaneous hypoglycaemia. It is clear that the basal dose must be "tailing off" in strength of action at night.

\section{Summary}

1. The chemical nature and physiological action of zinc protamine insulin is described.

2. We have used it as one dose a day, from 16 to 50 units, in forty-two insulin patients of all grades of severity. In all cases the urine passed twenty-four hours afterwards can be made sugar-free and the blood sugar normal or nearly so. In many severe cases hyperglycaemia and glycosuria after carbohydrate meals, especially breakfast, cannot be controlled by such a dose of zinc protamine insulin alone, but can be better regulated by the addition of a suitable dose of soluble insulin injected in the same syringe.

3. The adjustment of diet, the dosage of insulin, and the possibility and prevention of hypoglycaemia are discussed. We consider that careful study of each case is essential.

4. We conclude, so far as our limited experience goes, that most severe diabetes can be well controlled and the patient maintained in excellent health by one injection a day, either of zinc protamine insulin suspension alone or with the addition of some soluble insulin. The initial stages of such treatment should be carried out under close observation.

We wish to thank our patients for their keen co-operation, and the nursing staff and the dietetic and laboratory staff in our department for loyal assistance in the extra work which these studies have required. We are indebted to Professor Best of Toronto for our first supply of zinc protamine insulin and to Messrs. Boots Pure Drug Co. Ltd., who have supplied large quantities free since November, 1936.

\section{REFERENCES}

Fisher, A. M., and Scott, D. A. (1935). J. Pharm. exp. Therap., $55,206$.

Hagedorn, H. C., et al. (1936). J. Amer. med. Ass., 106, 177.

Joslin, E. P., et al. (1936). New Eng. J. Med., 214, 1079.

Krarup, N. B. (1935). Gad, Copenhagen.

Lawrence, R. D:, and Archer, N. (1936). British Medical Journal, $1,747$.

Rabinowitch, I. M., et al. (1936a). Canad. med. Ass. J., 35, 124. - (1936). Ibid., 35, 239.

Root, H. W., et al. (1936). J. Amer. med. Ass., 106, 180.

Scott, D. A., and Fisher, A. M. (1935). Biochem. J., 29, 1048.

Wilder, R. M. (1936). Proc. Mayo Clin., 11, 257.

M. Schubert (Derm. Wschr., December 19, 1936, p. 1653) believes with Miescher, Musger, and Kogoj that lichen sclerosus atrophicans represents an idiopathic affection, having the following characteristics: some spots are rounded or polygonal, of irregular but well-defined outlines. Their colour is bluish-white. They are either of the same level as the surrounding skin or slightly prominent or inversely slightly sunk. The surrounding skin is not red, but may show a slightly pigmented ring. Some of the spots develop into verrucous foci of grey-brownish colour with more or less extensive whitish margins. Small spots may coalesce to form large irregular but welldelimited foci of the colour of porcelain. There are also sunk, smooth, white, rounded scars with fine telangiectases. The affection can be distinguished both clinically and histologically from lichen ruber planus and from the circumscribed sclerodermia, although there are transitional forms between lichen sclerosus atrophicans and the lastnamed two affections. The author prefers to call the disease "lichen sclerosus atrophicans" rather than "white spot disease" (Weissflecken Krankheit).

\section{THE NOCIFENSOR SYSTEM OF NERVES AND ITS REACTIONS *}

BY

\author{
SIR THOMAS LEWIS, M.D., F.R.C.P., F.R.S. \\ Physician to University College Hospital, London
}

\section{LECTURE II}

The three reactions described in the first lecture were all discovered by observations carried out exclusively upon human subjects. The fourth, to which I now come, was first described in animals, and has been submitted to close experimental investigation in these.

\section{The "Antidromic" Flush}

The first definite statement of vasodilator nerves in the posterior roots was made by Stricker in 1876. But his observations remained unaccepted until Bayliss's careful experiments were recorded in 1901. Bayliss (1900-2) found that stimulation of the distal ends of the cut posterior roots by current, or mechanically, produces a long-lasting vasodilatation of the limb supplied. He showed that the cell stations of the fibres conducting the impulses to the vessels lie in the posterior root ganglia, for the effect remains after the posterior roots are cut, but is lost after the ganglia are excised. He concluded that the fibres concerned are the ordinary sensory afferent fibres of the posterior roots and that vasodilatation of the limb is effected by " antidromic" conduction, as he called it at Langley's suggestion. Similar vasodilatation can be produced by stimulating the distal ends of nerves to the limbs or cutaneous nerves, and these reactions have been studied by Langley (1923-4) and by myself and others (Lewis and Marvin, 1927), mainly in the cat's paw. Foerster has shown in recent years that reddening of the human skin over appropriate segments occurs when the posterior roots, or nerves of the limb running to the skin, have been cut and are stimulated distally. In the case of the cat stimulation of a small nerve of the paw flushes the bare surfaces of those pads which the particular nerve supplies, and flushes them sharply to a boundary. Langley first observed this, and, adopting my method of identifying active capillary dilatation, found that the flushing is produced even during circulatory arrest. He therefore, and rightly, concluded that the flush is due to capillary rather than to arteriolar dilatation in this animal.

The conception that the vasodilatation is due to antidromic conduction in sensory nerve fibres was grounded upon the contemporary belief that all posterior root fibres are afferent. Several workers (Ranson, Kuré, Tower) have since described intact fibres in the spinal ends of the cut posterior roots many days after section. These have been much discussed, and have generally been regarded as regenerated from the root ganglion. But Kahr and Sheehan (1933) traced intact fibres in this root after removing the ganglia; and Barron and Matthews (1934-5) have recently supplied some remarkable evidence, both physiological and anatomical, that fibres having their cell stations in the posterior root ganglia pass into the cord and send collaterals out again through neighbouring posterior roots on their way to the periphery. It seems from the observations of Matthews that these fibres normally carry impulses out to the periphery; but if that is so then it is difficult to regard the system of nerves in question as subserving a sensory function. Thus the evidence from this source inclines us to recognize that there are fibres other than sensory in the posterior

* The Hume Lectures delivered at Newcastle-on-Tyne, February 4 and 11. Lecture I was published last week. 